\section{Growing large and fast: is infant growth relevant for the early origins of childhood asthma?}

\author{
Liesbeth Duijts
}

Early growth seems to be important for the risk of respiratory diseases in childhood and adulthood. A recent meta-analysis reported that low birth weight is associated with impaired lung function and asthma in childhood, and that these associations are partly explained by gestational age at birth. ${ }^{1}$ Low birth weight seems also associated with impaired lung function and increased risks of asthma and chronic obstructive airway disease in adulthood. ${ }^{2}$ Although birth weight is an important early growth measure and may reflect early development, it has important limitations. Birth weight is the end point of fetal growth and the beginning of infant growth.

Different prospective cohort studies have recently been set up to disentangle the association of early growth with respiratory diseases at later ages. A prospective birth cohort study showed that a higher fetal crown-rump length in first trimester was associated with a lower risk of wheezing, asthma and higher lung volumes. ${ }^{3}$ Also, a greater abdominal circumference during second half of pregnancy was associated with lower risk of atopic wheezing, ${ }^{5}$ whereas a higher femur length in second trimester was associated with lower risk of asthma. ${ }^{3}$ However, when individual fetal growth characteristics were combined into estimated fetal weight, associations of fetal weight growth with childhood wheezing or asthma were not consistent. ${ }^{4} 67$ Restricted fetal weight growth was associated with higher respiratory resistance ${ }^{6}$ and lower $\mathrm{FEV}_{1}$ in childhood. ${ }^{4}$ Thus, these findings are in line with the findings using birth weight as early growth measure. Increased fetal growth from early pregnancy onwards seems to lead to

Divisions of Respiratory Medicine and Neonatology, Department of Pediatrics, Erasmus MC, University Medical Center Rotterdam, Rotterdam, the Netherlands

Correspondence to Dr Liesbeth Duijts, Erasmus MC - Sophia Children's Hospital, University Medical Center Rotterdam, Sp-3435; PO Box 2060, Rotterdam 3000 CB, the Netherlands; I.duijts@erasmusmc.nl lower risks of respiratory diseases at later ages.

Studies that examined the combined effects of growth in both fetal life and infancy are scarce, but suggest that increased infant growth is associated with an increased risk of asthma symptoms in preschool children. ${ }^{5}$ Most studies used weight measures for infant growth. To better understand how infant growth affects childhood asthma, knowledge is needed on more detailed and specific individual longitudinal infant weight and length growth measures. In Thorax, Popovic et $a l^{8}$ examined the associations of three biologically interpretable weight growth measures, including size, weight gain velocity and age at peak weight velocity, with wheezing among 4492 children up to age 18 months participating in the NINFEA study, an internet-based birth cohort. Results showed that size and weight gain velocity, but not age at peak weight velocity, were both independently associated with an approximately 1.3 -fold risk of wheezing between 6 and 12 months, and stronger when a more specific wheezing phenotype combined with asthma medication use as the outcome was used. Since wheezing in early life is not specific for asthma and could also be present in preterm or small for gestational age born infants with small airways, or in infants with respiratory tract infections, the authors performed sensitivity analyses excluding children born small for gestational age, or adjusted for respiratory tract infections. The results were not explained by these specific groups. Thus, their results suggest that growing large and fast in infancy could affect the risk of wheezing in early life.

In line with the study from Popovic et al, previous birth cohort studies showed that early persistent obesity ${ }^{9}$ or rapid weight growth in the first 2 years ${ }^{10}$ were associated with an up to twofold increased risk of asthma at school age. More specifically, rapid weight growth between 0 and 3 months seemed most consistently associated with increased risk of asthma, bronchial responsiveness and reversibility, and lower $\mathrm{FEV}_{1} / \mathrm{FVC}$ and $\mathrm{FEF}_{75} / \mathrm{FVC}$ ratios. ${ }^{11}$ Peak weight velocity was associated with an up to 1.3 -fold increased risk of wheezing or asthma up to age 10 years, and with lower lung function at 15 years, while peak height velocity seemed not associated with these outcomes. $^{12-15}$ The additional value of the current study is that size and velocity of infant weight growth, measured by a complex but carefully applied method, and their associations with wheezing were independently examined. This is important when studying underlying mechanisms for these associations, which might be different. Altogether, results of these studies strongly suggest that early infancy is a critical age window in which increased infant weight and weight gain velocity, possibly independent of fetal growth, could lead to the development of respiratory diseases at later ages.

The mechanisms by which infant growth affects respiratory outcomes at later ages are not well known. It has been suggested that adaptations related to infant growth and specifically alveolar growth, which partly appear in the first years after birth, affect the risk respiratory diseases. It could be hypothesised that the influence of increased infant weight on wheezing or asthma might be due to direct mechanical effects of intrathoracic and abdominal fat deposition leading to reduced pulmonary capacity or increased production of systemic proinflammatory mediators by fat tissue. ${ }^{16}$ Increased infant weight gain velocity could lead to dysanapsis, a dissociation between somatic and pulmonary growth, which subsequently might lead to wheezing.

An important limitation of the study by Popovic et al is that causality cannot be directly inferred because of the observational design. Also, size and weight gain velocity could affect wheezing but reversed causation may be present. A cross-lagged modelling approach, taking potential directions of associations into account, and further longitudinal follow-up studies with respiratory outcomes at older ages might address this issue. It also remains of interest whether it is infant growth that affects the risk of wheezing or whether endocrine or inflammatory factors related to infant growth are more relevant for the development of wheezing. Infant growth could affect body composition such as fat mass index, android to gynoid fat ratio and abdominal fat. ${ }^{17}$ Also, infant growth is mainly affected by child's diet. Detailed 
measurements of neonatal and infant body composition and diet might be better predictors for the development of wheezing. Infant growth is related to neonatal and fetal growth. The role of narrow or small airways in the neonatal period reflected by lower neonatal lung function ${ }^{18}$ and detailed fetal weight trajectories, including fetal weight velocity and gestational age at peak fetal weight velocity, and their relation with infant growth and wheezing need to be elucidated.

The NINFEA study has made a welcome addition to the literature and supports the concept of using more detailed infant growth measures to better understand the origins of respiratory diseases focused on growth. Further long-term follow-up studies are needed to support the reported findings. Furthermore, identification of the mechanisms underlying the associations of early growth with respiratory health and disease throughout the life course is urgently needed to develop preventive strategies focused on pregnant women and their young children.

Contributors LD contributed to the conception, drafted the article, revised it critically for important intellectual content and gave final approval of the version to be published.

Competing interests None declared.

Provenance and peer review Commissioned; externally peer reviewed.

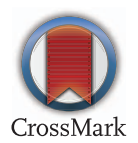

To cite Duijts L. Thorax 2016;71:1071-1072.

Published Online First 6 September 2016

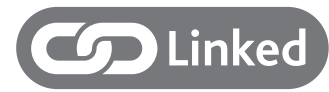

- http://dx.doi.org/10.1136/thoraxjnl-2015-208208

Thorax 2016;71:1071-1072.

doi:10.1136/thoraxjn-2016-209061

\section{REFERENCES}

1 den Dekker HT, Sonnenschein-van der Voort AM, de Jongste JC, et al. Early growth characteristics and the risk of reduced lung function and asthma: a meta-analysis of 25,000 children. J Allergy Clin Immunol 2016;137:1026-35.

2 Lawlor DA, Ebrahim S, Davey Smith G. Association of birth weight with adult lung function: findings from the British Women's Heart and Health Study and a meta-analysis. Thorax 2005;60:851-8.

3 Turner SW, Campbell D, Smith N, et al. Associations between fetal size, maternal \{alpha\}-tocopherol and childhood asthma. Thorax 2010;65:391-7.

4 Turner S, Prabhu N, Danielan P, et al. First- and second-trimester fetal size and asthma outcomes at age 10 years. Am J Respir Crit Care Med 2011;184:407-13.

5 Pike KC, Crozier SR, Lucas JS, et al. The Southampton Women's Survey Study Group. Patterns of fetal and infant growth are related to atopy and wheezing disorders at age 3 years. Thorax 2010;65:1099-106.

6 Sonnenschein-van der Voort AM, Gaillard R, de Jongste $\mathrm{JC}$, et al. Foetal and infant growth patterns, airway resistance and school-age asthma. Respirology 2016;21:674-82

7 Sonnenschein-van der Voort AM, Jaddoe VW, Raat $\mathrm{H}$, et al. Fetal and infant growth and asthma symptoms in preschool children: the Generation R Study. Am I Respir Crit Care Med 2012;185:731-7.
8 Popovic M, Pizzi C, Rusconi F, et al. Infant weight trajectories and early childhood wheezing: the NINFEA birth cohort study. Thorax 2016;71: 1091-6.

9 Ziyab AH, Karmaus W, Kurukulaaratchy RJ, et al. Developmental trajectories of Body Mass Index from infancy to 18 years of age: prenatal determinants and health consequences. J Epidemiol Community Health 2014;68:934-41.

10 Rzehak P, Wijga AH, Keil T, et al. Body mass index trajectory classes and incident asthma in childhood: results from 8 European Birth Cohorts-a Global Allergy and Asthma European Network initiative. J Allergy Clin Immunol 2013;131:1528-36.

11 Sonnenschein-van der Voort AM, Howe LD, Granell $R$, et al. Influence of childhood growth on asthma and lung function in adolescence. J Allergy Clin Immunol 2015;135:1435-43.e7.

12 Magnus MC, Stigum H, Håberg SE, et al. Peak weight and height velocity to age 36 months and asthma development: the Norwegian Mother and Child Cohort Study. PLOS ONE 2015;10: e0116362.

13 Flexeder C, Thiering E, Brüske I, et al. Growth velocity during infancy and onset of asthma in school-aged children. Allergy 2012;67:257-64.

14 Mebrahtu TF, Feltbower RG, Parslow RC. Effects of birth weight and growth on childhood wheezing disorders: findings from the Born in Bradford Cohort. BMJ Open 2015;5:e009553.

15 Claudia F, Thiering E, von Berg A, et al. Peak weight velocity in infancy is negatively associated with lung function in adolescence. Pediatr Pulmonol 2016;51:147-56.

16 Permaul P, Kanchongkittiphon W, Phipatanakul W. Childhood asthma and obesity - what is the true link? Ann Allergy Asthma Immunol 2014;113:244-6.

17 Gishti 0, Gaillard R, Manniesing R, et al. Fetal and infant growth patterns associated with total and abdominal fat distribution in school-age children. J Clin Endocrinol Metab 2014;99:2557-66.

18 Haland G, Carlsen KC, Sandvik L, et al. Reduced lung function at birth and the risk of asthma at 10 years of age. $N$ Engl J Med 2006;355:1682-9. 